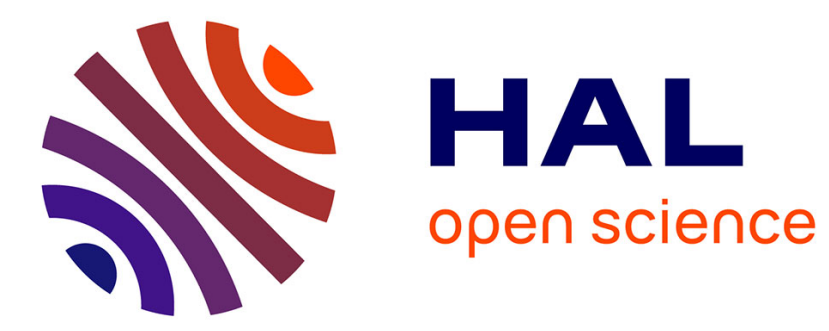

\title{
Inégalités et solidarités dans l'agriculture française
}

Jean-Marc Boussard

\section{To cite this version:}

Jean-Marc Boussard. Inégalités et solidarités dans l'agriculture française: Introduction. 1982, 152, pp.3-4. 10.3406/ecoru.1982.2908 . hal-02726712

\section{HAL Id: hal-02726712 \\ https://hal.inrae.fr/hal-02726712}

Submitted on 2 Jun 2020

HAL is a multi-disciplinary open access archive for the deposit and dissemination of scientific research documents, whether they are published or not. The documents may come from teaching and research institutions in France or abroad, or from public or private research centers.
L'archive ouverte pluridisciplinaire HAL, est destinée au dépôt et à la diffusion de documents scientifiques de niveau recherche, publiés ou non, émanant des établissements d'enseignement et de recherche français ou étrangers, des laboratoires publics ou privés.

\section{(이)(\$)}

Distributed under a Creative Commons Attribution - NonCommercial - NoDerivatives| 4.0 
J.M. Boussard

\section{Inégalités et solidarités dans l'agriculture française : introduction}

In: Économie rurale. $\mathrm{N}^{\circ} 152,1982$. pp. 3-4.

Citer ce document / Cite this document :

Boussard J.M. Inégalités et solidarités dans l'agriculture française : introduction. In: Économie rurale. №152, 1982. pp. 3-4.

doi : $10.3406 /$ ecoru.1982.2908

http://www.persee.fr/web/revues/home/prescript/article/ecoru_0013-0559_1982_num_152_1_2908 


\title{
«inégalités et solidarités»
}

\section{INTRODUCTION}

\author{
J.M. BOUSSARD
}

Peu de mots sont chargés d'un contenu émotionnel aussi grand que ceux qui définissent le thème choisi pour cette session de la SFER. En outre, la définition du programme s'est faite durant l'été et l'automne 1981, c'est-à-dire peu après le changement de majorité politique en France. Cela signifie que la tâche des organisateurs n'a pas été facile, beaucoup de propositions de communication étant plus dictées par le souci d'apporter de l'eau au moulin dc tclle ou telle tendance politique que par celui de l'analyse économique sereine et objective. Peut-être du reste, est-il impossible d'aborder de telles questions de façon détachée. La recherche d'un approfondissement réel des connaissances a pourtant été le souci principal du comité du programme (1).

La séance d'ouverture est traditionnellement dans les sessions de la SFER, l'occasion d'inviter des personnalités extérieures au cercle restreint (du reste très relativement) des économistes ruraux. En raison des implications politiques du thème, il a été décidé cette fois de demander l'intervention des personnes engagées dans l'action politique et syndicale, à savoir Pierre CORMORECHE et Bernard THAREAU, dont on lira ci-après le témoignage. En même temps, il a paru nécessaire de demander la participation de spécialistes de la mesure des inégalités, en l'occurence les membres de l'état-major d'une nouvelle direction de l'INSEE, destiné précisément à fournir des statistiques sur cette question. La hauteur de leurs vues, la mise en évidence de toutes les difficultés conceptuelles liées à la mesure des inégalités sociales, la rigueur d'une analyse qui ne partait du sens commun que pour en montrer les insuffisances, tout cela a été particulièrement apprécié des participants à la session.

Au contraire de la séance d'ouverture, plus synthétique, les séances simultanées sont plutôt destinées à la présentation de résultats de recherches. Le problème des inégalités et des solidarités pouvant être approché de plusieurs points de vue, il ne faut pas s'étonner de la diversité des méthodes et des résultats, d'autant plus que la coexistence des deux thèmes «inégalités» et "solidarités» ne poussait pas au monolithisme. Une question préalable se posait du reste à ce sujet : fallait-il laisser chaque auteur s'exprimer sur les deux sous-thèmes, ou isoler en quelque sorte le sous-thème solidarité dans une session spéciale ? Dans la mesure où les deux choses sont évidemment liées, la première solution semblait devoir s'imposer. Mais en raison de la tendance dominante à insister sur les inégalités, il a semblé que le seul moyen de parler réellement des solidarités consistait au contraire à regrouper dans une des sessions les communications qui les concernaient directement. On a pu constater ainsi que si la solidarité entre agriculteurs est un phénomène indiscutable et important (comme l'ont montré MM. NOUYRIT et ARNAUD) il était encore peu étudié, et que les concepts associés sont encore assez flous. Par ailleurs, M. FERTE et Mme ALLAUME soulignent l'ampleur des solidarités involontaires, dues au système de prélèvements sociaux, et montrent le danger de n'avoir plus rien à redistribuer.

Le volet inégalités, en revanche, a pu être examiné sous de nombreux angles. La première chose à faire est évidemment le constat statistique. Avec des points de vues différents, M. PORTIER, MM. GIRARD et PROST, et M. BUTAULT s'efforcent d'inventorier les sources disponibles et de démontrer qu'elles peuvent être interprétées dans des sens différents. Dans le même esprit, il est intéressant d'étudier l'évolution de longue période. Cela ne peut guère se faire qu'au niveau régional. C'est l'objet des communications de M. LEVY LEBOYER (qui se place dans une perspective séculaire) et de M. BONNIEUX (dont l'horizon est de l'ordre de la décennie).

Les inégalités étant constatées il faut examiner comment elles sont vécues par les intéressés. Plusieurs contributions abordent cette question. M. GRIGNON insiste sur la notion de "professionnalisme» qui permet d'interpréter et d'expliquer le comportement des agriculteurs vis-à-vis d'inégalités qui ne sont pas forcément ressenties comme des injustices. M. BLANC cherche à jeter les bases d'une typologie des exploitants en fonction de leur situation sociale Mme EIZNER, en analysant le discours des organisations professionnelles, essaie de définir l'image que ces dernières cherchent à donner de la question pour le grand public - tout en soulignant les aspects éventuellement contradictoires de cette image. M. REMY a montré l'importance du système de récompense social (qui n'est pas nécessairement monétaire) dans la diffusion des innovations, et la sélection des «meilleurs» exploitants.

Il ne suffit pas de mesurer les inégalités d'un exploitant à l'autre, ou d'une région à l'autre. Il faut aussi voir ce qui se passe à l'intérieur même de l'exploitation. C'est le point de vue de Mmes BARTHEZ et de SEVERAC, pour les rapports entre hommes et femmes, de même que celui de $M$. ALLAIRE, qui a insisté sur la variété des statuts sociaux des «travailleurs de la terre».

Il reste à expliquer le phénomène des inégalités et à rechercher les moyens d'agir sur la répartition. Le facteur personnel entre-t-il en ligne de compte? En d'autres termes, $y$-a-t-il de bons (riches) ou de mauvais (pauvres) agriculteurs ? MM. BOURLIAUD et COLLOMB ont tenté de répondre à cette question, l'un à partir d'études sur modèle, l'autre à partir de l'observation des migrations dans une petite région du midi. Tous deux insistent sur l'importance des facteurs «objectifs» dans l'étiologie de la pauvreté agricole. 
Peut-on aussi faire intervenir le facteur culturel dans l'explication des inégalités ? Les chiffres fournis à ce sujet par M. JEGOUZO montrent de quelle façon les enfants des paysans pauvres sont désavantagés dans la recherche d'une position sociale.

Les actions pour réduire les inégalités sont-elles efficaces ? D'un point de vue général, si la communication prévue sur les effets de la politique agricole par $M$. de MARTY a dû être retirée au dernier moment en raison des nouvelles fonctions de l'auteur au cabinet du ministre des finances, MM. HAIRY et PERRAUD, en traitant du cas particulier du prix du lait dans l'Ouest, ont montré sur un exemple que les effets pervers d'une politique pouvaient l'emporter sur ses effets directs. D'un point de vue moins général, mais sans doute tout aussi central, on pouvait s'attacher au rôle spécifique du crédit. Celui-ci réduit-il ou amplifie-t-il les inégalités? Tandis que M. NEVEU pense que le crédit a surtout permis le maintien des exploitations familiales, MM. BLOGOWSKI, BOMPARD et al. ont insisté sur les difficultés financières des exploitations trop endettées.

Il est trop évident après ce bref survol du programme, que celui-ci n'a pas traité le sujet dans toute son ampleur. Les résultats de recherche peuvent rarement faire autre chose que donner un coup de projecteur sur tel ou tel aspect particulier, sans presque jamais pouvoir éclairer l'ensemble de la scène. L'agriculture française est-elle maintenant plus ou moins égalitaire, plus ou moins solidaire que du temps de Louis XIV ? Si elle est plus égalitaire, quel prix a-t-il fallu payer pour cela ? Faut-il choisir entre l'efficacité et l'inégalité ? Dans quelles mesures les solidarités sont-elles plus importantes que les inégalités? Les contributions présentées ne permettaient pas de répondre à ces questions.

Il faut enfin préciser à nos lecteurs que le manque de place a contraint les rédacteurs de la revue à renoncer à publier certaines des contributions présentées lors de la session. Le travail de M. PORTIER à été publié dans les Cahier de Statistiques Agricoles (avril 1982). Ceux de MM. NEVEU, BOURLIAUD et COLLOMB feront sans doute l'objet d'une publication plus développée dans un numéro ultérieur de cette revue. Les autres communications qui figuraient dans le programme et qui sont absentes de ce numéro se prêtaient mal à la publication soit à cause de leur longueur, soit parce qu'elles reprenaient des idées déjà développées ailleurs, soit enfin, parce que tout en apportant un éclairage nouveau sur certains problèmes, il est apparu au comité de rédaction qu'elles étaient moins importantes que les autres, et devaient dont être sacrifiées à la contrainte du nombre de pages maximum autorisé pour ce numéro par le budget de notre société. 